\title{
Scolecobasidium terreum
}

National Cancer Institute

\section{Source}

National Cancer Institute. Scolecobasidium terreum. NCI Thesaurus. Code C127745.

A species of soil-borne, saprotrophic fungi in the phylum Ascomycota. S. terreum is the type species for Scolecobasidium. 